TRANSACTIONS OF THE

AMERICAN MATHEMATICAL SOCIETY

Volume 350, Number 5, May 1998, Pages 1913-1930

S 0002-9947(98)01976-X

\title{
LINKAGE AND SUMS OF IDEALS
}

\author{
MARK R. JOHNSON
}

\begin{abstract}
It is shown (under mild conditions) that the sum of transversal ideals in a regular local ring cannot lie in the linkage class of a complete intersection. For a sum of geometrically linked Cohen-Macaulay ideals, we compute the depths of the conormal module and the first Koszul homology. As applications, we construct general examples of ideals which are strongly CohenMacaulay, strongly nonobstructed but not in the linkage class of a complete intersection, and Gorenstein ideals which are strongly nonobstructed but not syzygetic.
\end{abstract}

\section{INTRODUCTION}

If $R$ is a local Gorenstein ring, two $R$-ideals $I$ and $J$ are said to be linked (written $I \sim J)$ if there is a regular sequence $\underline{\alpha}=\alpha_{1}, \ldots, \alpha_{g}$ in $I \cap J$ with $J=(\underline{\alpha}): I$ and $I=(\underline{\alpha}): J$. This notion is due to Peskine and Szpiro [21], although it was essentially known classically. It turns out to be a very useful approach towards classifying ideals (and algebraic varieties). Indeed, we obtain an equivalence relation by declaring that $I$ and $J$ belong to the same linkage class when there is a sequence of links

$$
I=I_{0} \sim I_{1} \sim \cdots \sim I_{n}=J
$$

joining $I$ and $J$. From this point of view, the simplest ideals are the licci ideals, those in the linkage class of a complete intersection; they include all perfect ideals of grade 2 ([1], [5]), and all perfect Gorenstein ideals of grade 3 ([33]). Thus licci ideals are seen to generalize these well-known examples to higher codimensions. Moreover, due to the work of various authors ([3], [4], [6], [8], [10], [11], [13], [14], [15], [18], [26], [28]), most notably Huneke and Ulrich, licci ideals enjoy some very good properties. For instance, any licci ideal is strongly Cohen-Macaulay ([8]) and strongly nonobstructed $([3],[4])$. (We refer the reader to Section 1 for any undefined terminology.) Individually, these properties are important in the study of blowing-up rings ([7]), and in deformation theory ([3], [6]). Despite this fact, most of the known examples of strongly nonobstructed ideals, or ideals say whose entire linkage class is strongly Cohen-Macaulay, happen to be licci. It is of interest to obtain additional methods of producing strongly Cohen-Macaulay or strongly nonobstructed ideals to which one may apply the aforementioned theories. There is also the question as to what extent are licci ideals possibly characterized by these properties.

In trying to discover new ideals, it is natural to consider a sum $I+J$ of two ideals, $I$ and $J$, having the requisite properties. In this work, we consider two

Received by the editors June 10, 1996.

1991 Mathematics Subject Classification. Primary 13C40, $13 \mathrm{C} 14$.

(C)1998 American Mathematical Society 
important cases when the sum is known to be Cohen-Macaulay whenever the ideals in question are:

(i) $I$ and $J$ are transversal (and $R$ is regular),

(ii) $I$ and $J$ are geometrically linked ideals.

In case (i), Huneke [9] used the vanishing of Tor to obtain a Künneth formula for the Koszul homology of $I+J$ in terms of that of $I$ and $J$. In particular, if $I$ and $J$ are transversal and are both strongly Cohen-Macaulay, then so is $I+J$. Similar remarks hold for the property of being strongly nonobstructed. Thus, if we take $I$ and $J$ to be transversal licci ideals, then the sum $I+J$ is both strongly CohenMacaulay and strongly nonobstructed. (The prototypical example of a transversal sum to bear in mind is the defining ideal of the product of the two affine varieties.) We are able to show the surprising result that this ideal is, however, essentially never licci: If $I$ and $J$ are transversal licci ideals, then $I+J$ is licci only if either $I$ or $J$ is a complete intersection (Theorem 2.6). This method produces the first known examples of nonlicci ideals which have these good homological properties only previously observed for the class of licci ideals. For example, we obtain, in a regular local ring $R$, strongly nonobstructed prime ideals of grade 5 whose entire linkage class is strongly Cohen-Macaulay, and strongly Cohen-Macaulay Gorenstein primes $I$ of grade 6 with $\operatorname{Tor}_{\bullet}^{R}(R / I, R / I)$ Cohen-Macaulay, which are not licci. It thus appears that licci ideals cannot be easily characterized by these sorts of homological properties.

In case (ii), where $I$ and $J$ are geometrically linked Cohen-Macaulay ideals, Peskine and Szpiro [21] observed that the sum $K=I+J$ is a Gorenstein ideal with grade $K=$ grade $I+1$. This is a nontrivial way to produce Gorenstein ideals from Cohen-Macaulay ones; it was essentially used by Kustin and Miller [17] in their study of Gorenstein ideals of grade 4, and more recently was studied in its own right by Ulrich [26]. Unlike the previous case (i) however, $K$ is licci whenever $I$ is $([26,2.1])$. It is still interesting to know when $K$, if not licci, is strongly Cohen-Macaulay and/or strongly nonobstructed (the latter in this case being just the Cohen-Macaulayness of the conormal module $K / K^{2}$ ). In [26], Ulrich proves that $K / K^{2}$ and $H_{1}(K)$ are Cohen-Macaulay whenever $I$ is strongly nonobstructed and $H_{1}(I)$ and $H_{1}(J)$ are Cohen-Macaulay. It is often useful, however, to relax the Cohen-Macaulayness of the Koszul homology to a weaker depth estimate on the powers of the ideal. Moreover, certain depth conditions force many of the important consequences of the strongly Cohen-Macaulay property ([27]). We are able to compute the depth of the modules $K / K^{2}$ and $H_{1}(K)$ directly in terms of the depths of corresponding modules of the linked ideals $I$ and $J$ (Theorem 3.3). As one application of this result, we are able to easily construct in abundance, in any grade at least 5 , Gorenstein prime ideals which are strongly nonobstructed, but not syzygetic. One such example is given in [26], but our method also makes clear why such examples should exist. Finally, we apply these results (in Example 3.10) to verify that a well-known example already had this property.

The results of this paper were obtained (in a slightly weaker form) in the author's Ph.D. dissertation at Michigan State University. He is grateful to his advisor Bernd Ulrich for his guidance.

\section{BACKGROUND ON LINKAGE}

In this section we recall some basic notions and results that we will use throughout the paper. 
Let $R$ be a Noetherian local ring and let $I$ be an (proper) $R$-ideal. By $\nu(I)$ we denote the minimal number of generators of $I$, and $d(I)=\nu(I)$ - grade $I$ is the deviation. If $d(I)=0(d(I) \leq 1)$, then $I$ is a complete intersection (an almost complete intersection, respectively). One says that $I$ satisfies $\left(C I_{k}\right)$ if $I_{p}$ is a complete intersection for every $p \in V(I)$ with $\operatorname{dim}(R / I)_{p} \leq k$, and that $I$ satisfies $G_{\infty}$ if $\nu\left(I_{p}\right) \leq \operatorname{dim} R_{p}$ for every $p \in V(I)$. An ideal is generically a complete intersection if it is unmixed and satisfies $\left(C I_{0}\right)$. For an $R$-module $M$, we let $S_{i}(M)$ denote the $i$-th symmetric power of $M$, and let $\tau$ denote the torsion submodule of $M$. If $X$ denotes a finite set of variables over $R, R[X]$ denotes the polynomial ring over $R$, while $R(X)=R[X]_{m R[X]}$, where $m$ is the maximal ideal of $R$.

Let $R$ be a local Cohen-Macaulay ring. An ideal $I$ is Cohen-Macaulay or Gorenstein if $R / I$ has this property, and is perfect if it is Cohen-Macaulay and has finite projective dimension. We denote by $\omega_{R}$ the canonical module of $R$, and by $r(R)$ the type of $R$.

Definition 1.1 ([21]). Let $R$ be a (not necessarily local) Gorenstein ring and let $I$ and $J$ be $R$-ideals.

(a) $I$ and $J$ are linked, written $I \sim J$, if there is an $R$-regular sequence $\underline{\alpha}=$ $\alpha_{1}, \ldots, \alpha_{g}$ in $I \cap J$ such that $J=(\underline{\alpha}): I$ and $I=(\underline{\alpha}): J$.

(b) $I$ and $J$ are geometrically linked if $I$ and $J$ are linked and ht $I+J>g$.

It follows that two linked ideals are unmixed and of the same grade $g$. In addition, $I$ and $J$ are geometrically linked if and only if they are linked and have no common minimal primes. In this case one has that $I \cap J=(\underline{\alpha})$, i.e., $V(\underline{\alpha})$ is the schemetheoretic union of $V(I)$ and $V(J)$, and hence that $\underline{\alpha}$ generates $I$ and $J$ generically; in particular, $I$ and $J$ are generically a complete intersection.

Proposition $1.2([21])$. Let $R$ be a (not necessarily local) Gorenstein ring, let I be a unmixed $R$-ideal, let $\underline{\alpha}$ be a maximal regular sequence with $(\underline{\alpha}) \varsubsetneqq I$ and put $J=(\underline{\alpha}): I$.

(a) $I=(\underline{\alpha}): J$ (i.e., $I$ and $J$ are linked).

(b) $I$ is Cohen-Macaulay (perfect) if and only if $J$ is Cohen-Macaulay (perfect).

(c) Let $R$ be local and I be Cohen-Macaulay. Then $\omega_{R / I} \cong J /(\underline{\alpha})$. In particular, $r(R / I)=\nu(J /(\underline{\alpha}))$.

Definition 1.3. Let $R$ be a local Gorenstein ring.

(a) Two $R$-ideals $I$ and $J$ are in the same linkage class if there exists a sequence of links $I=I_{0} \sim I_{1} \sim \cdots \sim I_{n}=J$. If $n$ is even, $I$ and $J$ are in the same even linkage class (or are evenly linked). If $n=2$, then $I$ and $J$ are doubly linked.

(b) An $R$-ideal is licci if it is in the linkage class of a complete intersection ideal.

Note that by Proposition 1.2b, licci ideals are perfect. We recall some important invariants of the linkage class.

Let $I=\left(f_{1}, \ldots, f_{n}\right)$ be an ideal. By $H_{i}(I)$ we denote the $i$-th homology module of the Koszul complex $K_{\bullet}\left(f_{1}, \ldots, f_{n}\right)$. For an integer $k$, the property that $H_{i}(I)$ is Cohen-Macaulay for $0 \leq i \leq k$ is independent of the generating set $([8,1.5])$.

Definition $1.4([9])$. Let $R$ be a local Cohen-Macaulay ring. An $R$-ideal $I$ is strongly Cohen-Macaulay if $H_{i}(I)$ is Cohen-Macaulay for every $i$.

Lemma 1.5 ([8]). Let $R$ be a local Cohen-Macaulay ring, let $I=\left(f_{1}, \ldots, f_{n}\right)$ be an $R$-ideal with $f_{1} R$-regular, and let "-_" denote reduction modulo $f_{1}$. 
(a) There is an exact sequence

$$
0 \rightarrow H_{i}(I) \rightarrow H_{i}(\bar{I}) \rightarrow H_{i-1}(I) \rightarrow 0
$$

for every $i \geq 0$.

(b) $I$ is strongly Cohen-Macaulay if and only if $\bar{I}$ is strongly Cohen-Macaulay.

A weaker condition on the Koszul homology is also useful. We say that $I$ is syzygetic if the natural map $S_{2}(I) \rightarrow I^{2}$ is an isomorphism, or equivalently if the natural sequence

$$
0 \rightarrow H_{1}(I) \rightarrow \oplus R / I \rightarrow I / I^{2} \rightarrow 0
$$

is exact $([22,1.2])$. It follows that if $I$ is generically a complete intersection, then $I$ is syzygetic if and only if $H_{1}(I)$ is $R / I$-torsion free.

Definition $1.7([6])$. Let $R$ be a local Gorenstein ring. An $R$-ideal $I$ is strongly nonobstructed if $I / I^{2} \otimes_{R / I} \omega_{R / I}$ is Cohen-Macaulay.

Strong Cohen-Macaulayness plays an important role in the theory of blowingup rings ([7]) and in the study of residual intersections ([9], [13]). On the other hand, the notion of being strongly nonobstructed arises from deformation theory ([3], [6]): If the $R$-ideal $I$ is strongly nonobstructed and generically a complete intersection, then one obtains the vanishing of the cotangent cohomology $T^{2}((R / I) / R) \cong \operatorname{Ext}_{R / I}^{1}\left(I / I^{2}, R / I\right) \cong \operatorname{Ext}_{R / I}^{1}\left(I / I^{2} \otimes_{R / I} \omega_{R / I}, \omega_{R / I}\right)$.

Theorem $1.8([8])$. Let $R$ be a local Cohen-Macaulay ring and let $I$ and $J$ be $R$ ideals which are evenly linked. Then $I$ is strongly Cohen-Macaulay if and only if $J$ is strongly Cohen-Macaulay.

Theorem 1.9 ([3], [4]). Let $R$ be a local Gorenstein ring, and let $I$ and $J$ be linked $R$-ideals. Suppose that either I is perfect or that I is Cohen-Macaulay and the link $I \sim J$ is geometric. Then $I$ is strongly nonobstructed if and only if $J$ is strongly nonobstructed.

(A proof in the geometric case also appears in the recent book [30, 4.2.12].)

The two previous results thus imply that any licci ideal is strongly nonobstructed and (its entire linkage class is) strongly Cohen-Macaulay (as any complete intersection is linked to itself). Linkage provides an abundance of examples of ideals having these properties. We will produce some new ones in the next section. We will need some further notions.

Definition 1.10 ([11]). Let $(R, I)$ and $(S, J)$ be pairs, where $R$ and $S$ are Noetherian local rings and $I \subset R, J \subset S$ are ideals, or $I=R$ or $J=S$.

(a) $(R, I)$ and $(S, J)$ are isomorphic, written $(R, I) \cong(S, J)$, if there is an isomorphism $\varphi: R \rightarrow S$ with $\varphi(I)=J$.

(b) $(S, J)$ is a deformation of $(R, I)$ if there is a sequence $\underline{x}=x_{1}, \ldots, x_{n}$ in $S$, regular on $S$ and $S / J$, such that $(S /(\underline{x}), J+(\underline{x}) /(\underline{x})) \cong(R, I)$.

(c) $(S, J)$ is essentially a deformation of $(R, I)$ if there is a sequence of pairs $\left(S_{i}, J_{i}\right), 1 \leq i \leq n$, with $\left(S_{1}, J_{1}\right)=(R, I),\left(S_{n}, J_{n}\right)=(S, J)$, such that for $1 \leq i \leq$ $n-1$, one of the following conditions is satisfied:

(i) $\left(S_{i+1}, J_{i+1}\right)=\left(\left(S_{i}\right)_{p},\left(J_{i}\right)_{p}\right)$ for some $p \in \operatorname{Spec} S_{i}$;

(ii) $\left(S_{i+1}, J_{i+1}\right)$ is a deformation of $\left(S_{i}, J_{i}\right)$;

(iii) $\left(S_{i+1}(X), J_{i+1} S_{i+1}(X)\right) \cong\left(S_{i}(Y), J_{i} S_{i}(Y)\right)$ for some finite sets of variables $X$ over $S_{i+1}$ and $Y$ over $S_{i}$. 
Lemma 1.11 ([14, proof of 2.1]). Let $R$ be a local Cohen-Macaulay ring, let $I$ be an $R$-ideal which is generically a complete intersection, let $(S, J)$ be a deformation of $(R, I)$ and let $k$ be an integer. Then $H_{i}(I)$ is Cohen-Macaulay for $0 \leq i \leq k$ if and only if $H_{i}(J)$ is Cohen-Macaulay for $0 \leq i \leq k$. In particular, $I$ is strongly Cohen-Macaulay if and only if $J$ is strongly Cohen-Macaulay.

The next result shows that links deform well. In particular, a deformation of a licci ideal is licci:

Lemma 1.12 ([11, 2.16]). Let $R$ be a local Gorenstein ring, let $I$ be a CohenMacaulay $R$-ideal, let $I=I_{0} \sim I_{1} \sim \cdots \sim I_{n}$ be a sequence of links, and let $(S, J)$ be a deformation of $(R, I)$. Then there is a sequence of links $J=J_{0} \sim \cdots \sim J_{n}$ in $S$ such that $\left(S, J_{i}\right)$ is a deformation of $\left(R, I_{i}\right)$ for every $0 \leq i \leq n$.

We define next the most general link of an ideal.

Definition 1.13 ([11]). Let $R$ be a Gorenstein ring, let $I$ be an unmixed $R$-ideal of grade $g>0$, and let $f_{1}, \ldots, f_{n}$ be any generating set of $I$. Let $X$ be a generic $g \times n$ matrix of variables over $R$ and let $\underline{\alpha}=\alpha_{1}, \ldots, \alpha_{g}$ denote the $R[X]$-regular sequence defined by $(\underline{\alpha})^{t}=X \cdot(\underline{f})^{t}$. Then $L_{1}(\underline{f})=(\underline{\alpha}) R[X]: I R[X]$ is the first generic link of $I$.

The first generic link $L_{1}(f)$ (or rather, the pair $\left.\left(R[X], L_{1}(f)\right)\right)$ is independent of the generating set, up to isomorphism of pairs (after adjoining variables) ([11, 2.11]). Thus we write $L_{1}(I)=L_{1}(f)$ and hence we may iterate this process and define the $i$-th generic link $L_{i}(I)$ by $\bar{L}_{i}(I)=L_{1}\left(L_{i-1}(I)\right)$ for $i \geq 1$. (We set $L_{0}(I)=I$.) Hence we obtain a canonical sequence of links $I S \sim L_{1}(I) S \sim \cdots \sim L_{n}(I) S$ in some polynomial extension $S$ of $R$. In addition, if $I$ is generically a complete intersection, then the link $I R[X] \sim L_{1}(I)$ is geometric $([10,2.5])$.

The following theorem shows that generic links are the prototypical sequence of links:

Theorem 1.14 ([11, 2.17]). Let $(R, m)$ be a local Gorenstein ring, let I be a CohenMacaulay $R$-ideal with grade $I>0$ and let $I=I_{0} \sim I_{1} \sim \cdots \sim I_{n}$ be a sequence of links in $R=R_{0}$. For $1 \leq i \leq n$, let $L_{i}(I) \subset R_{i}$ be $i$-th generic links, where $R_{i}$ is a polynomial ring over $R_{i-1}$, and put $S=R_{n}$. Then there exists a prime ideal $q$ in $S$ with $m \subset q$, such that $\left(S_{q}, L_{i}(I) S_{q}\right)$ is a deformation of $\left(R, I_{i}\right)$ for $1 \leq i \leq n$.

Finally, we will need to utilize one of the main results of [11], which gives a local structure theorem for licci ideals. We actually use a slightly improved version in [25]:

Theorem 1.15 ([25, 2.6]). Let $R$ be a regular local ring and let $I$ be a licci $R$-ideal which is not a complete intersection.

(a) If $I$ is Gorenstein, then $(R, I)$ has essentially a deformation $\left(S_{1}, J_{1}\right)$ with $S_{1} / J_{1} \cong\left(P[X] / P f_{4}(X)\right)_{\left(m_{p}, X\right)}$, where $P$ is a regular local ring, and $X$ is a generic alternating $5 \times 5$ matrix.

(b) If I is not Gorenstein, then $(R, I)$ has essentially a deformation $\left(S_{2}, J_{2}\right)$ with $S_{2} / J_{2} \cong\left(P[X] / I_{2}(X)\right)_{\left(m_{p}, X\right)}$, where $P$ is a regular local ring and $X$ is a generic $2 \times 3$ matrix.

\section{Sums of TRANSVERSAL IDEALS}

In this section $R$ denotes a regular local ring containing its residue field $k$. Recall that two $R$-ideals $I$ and $J$ are transversal if $\operatorname{Tor}_{1}^{R}(R / I, R / J)=0$, i.e., if $I \cap J=I J$. 
By the vanishing of Tor ([2] or [19]), the sum $I+J$ is then a Cohen-Macaulay ideal whenever $I$ and $J$ are.

Now if $R$ is complete, i.e., $R=k\left[\left[x_{1}, \ldots, x_{n}\right]\right]$, then the pair $(R, I+J)$ has a deformation $(S, \widetilde{I}+\widetilde{J})$, where $S=R\left[\left[y_{1}, \ldots, y_{n}\right]\right], \widetilde{I}=I S$, and $\widetilde{J}$ is extended from $k\left[\left[y_{1}, \ldots, y_{n}\right]\right]$. In this way, we are essentially reduced to studying the complete tensor product $R / I \widehat{\otimes}_{k} R / J$ of the complete local $k$-algebras $R / I$ and $R / J$.

We begin with an auxiliary construction, which will be useful in studying the linkage class of a transversal sum.

Definition 2.1. Let $I \subset k[[X]]$ and $J \subset k[[Y]]$ be unmixed ideals and let $\underline{\alpha}$ be a maximal regular sequence inside $I$, and let $\beta$ be a maximal regular sequence inside $J$, with $(\underline{\alpha}) \varsubsetneqq I$ and $(\underline{\beta}) \varsubsetneqq J$. The $R=k[[\bar{X}, Y]]$-ideal $\mathcal{L}=(\underline{\alpha}, \underline{\beta}) R:(I R+J R)$ is a transversal link of $I$ and $J$.

By Proposition $1.2 \mathrm{a}, \mathcal{L}$ is linked to $I R+J R$ by the sequence $\underline{\alpha}, \underline{\beta}$. In particular, any two transversal links of $I$ and $J$ are doubly linked, and thus share any property preserved under even linkage. Thus we will usually ignore the dependency of the transversal link on the chosen sequences, and write $\mathcal{L}$ for some transversal link of $I$ and $J$.

Remark 2.2. With the notation of Definition 2.1, if $I$ and $J$ are generically a complete intersection, then the link $I R+J R \sim \mathcal{L}$ may be chosen to be geometric.

Proof. As $I$ and $J$ are generically a complete intersection, there exist geometric links $I \stackrel{\alpha}{\sim} I_{1}$ in $k[[X]]$ and $J \stackrel{\beta}{\sim} J_{1}$ in $k[[Y]]$, thus ht $I+I_{1}>$ ht $I$ and ht $J+J_{1}>$ ht $J$. But

$$
\begin{aligned}
\mathcal{L} & =(\underline{\alpha}, \underline{\beta}) R:(I R+J R) \\
& =(\underline{\alpha}, \underline{\beta}) R: I R \cap(\underline{\alpha}, \underline{\beta}) R: J R \\
& =((\underline{\alpha}) R: I R, \underline{\beta}) \cap(\underline{\alpha},(\underline{\beta}) R: J R) \\
& =(((\underline{\alpha}): I) R, \underline{\beta}) \cap(\underline{\alpha},((\underline{\beta}): J) R) \\
& =\left(I_{1} R, \underline{\beta}\right) \cap\left(\underline{\alpha}, J_{1} R\right) \\
& =(\underline{\alpha}, \underline{\beta}) R+I_{1} R \cdot J_{1} R .
\end{aligned}
$$

It follows that the sum $(I R+J R)+\mathcal{L}=I R+J R+I_{1} R \cdot J_{1} R$ has height $\geq$ $\min \left\{\mathrm{ht}\left(I+I_{1}\right)+\right.$ ht $J, \mathrm{ht}\left(J+J_{1}\right)+$ ht $\left.I\right\}>$ ht $I R+J R$, and thus the link $I R+J R \sim \overline{\mathcal{L}}$ is geometric.

We next remark on the number of generators of any transversal link.

Remark 2.3. With the assumption of Remark 2.2, assume in addition that $I$ and $J$ are Cohen-Macaulay. Then $d(\mathcal{L})=r(R / I R) \cdot r(R / J R)$.

Proof. As $r(R / I R+J R)=r(R / I R) \cdot r(R / J R)$, the result follows from Proposition $1.2 \mathrm{c}$ once we have shown that $d(\mathcal{L})=\nu(\mathcal{L} /(\underline{\alpha}, \underline{\beta}))$. But if $m$ denotes the maximal ideal of $R$, this holds since $(\underline{\alpha}, \underline{\beta})+m L / m L \cong(\underline{\alpha}, \underline{\beta}) /\left(m(\underline{\alpha}, \underline{\beta})+(\underline{\alpha}, \underline{\beta}) \cap m I_{1} R\right.$. $\left.J_{1} R\right)=(\underline{\alpha}, \underline{\beta}) / m(\underline{\alpha}, \underline{\beta})$, as $(\underline{\alpha}, \underline{\beta}) \cap m I_{1} R \cdot J_{1} R \subset(\underline{\alpha}, \underline{\beta}) \cap I_{1} R \cdot \bar{J}_{1} R=(\underline{\alpha}, \underline{\beta}) \cap I_{1} R \cap$ $J_{1} R=(\underline{\alpha}) \overline{J_{1}} R+(\underline{\beta}) \bar{I}_{1} R \subset m(\underline{\alpha}, \underline{\beta})$.

The next result contains the crucial properties of the transversal link that we will need. 
Lemma 2.4. Let $I \subset k\left[\left[x_{1}, \ldots, x_{n}\right]\right]$ and $J \subset k\left[\left[y_{1}, \ldots, y_{n}\right]\right]$ be Cohen-Macaulay ideals which are generically a complete intersection, and let $\mathcal{L}$ be a geometric transversal link of $I$ and $J$.

(a) If $I$ and $J$ are linked to ideals whose first Koszul homology $H_{1}$ is CohenMacaulay, then $\operatorname{depth} R / \mathcal{L}^{2} \geq \operatorname{dim} R / \mathcal{L}-1$.

(b) If $I$ and $J$ are not Gorenstein, then $\mathcal{L}$ is not syzygetic.

(c) If I is Gorenstein and $J$ is geometrically linked to a strongly Cohen-Macaulay ideal $J_{1}$ with $J+J_{1} / J$ satisfying $G_{\infty}$, then $\mathcal{L}$ is strongly Cohen-Macaulay.

Proof. Let $(\underline{\alpha}) \subset I$ and $(\underline{\beta}) \subset J$ be the regular sequences used to define $\mathcal{L}$, and put $I_{1}=(\underline{\alpha}): I$ and $J_{1}=(\bar{\beta}): J$. Then $I \sim I_{1}$ and $J \sim J_{1}$ (Proposition 1.2a), and $\mathcal{L}=(\underline{\alpha}, \beta) R+I_{1} R \cdot J_{1} R$. We will denote by "-." reduction modulo the ideal $(\underline{\alpha}, \beta)$.

We first show (a). By assumption, $I_{1}$ and $J_{1}$ are doubly linked to ideals with $H_{1}$ Cohen-Macaulay, hence $H_{1}\left(I_{1}\right)$ and $H_{1}\left(J_{1}\right)$ are Cohen-Macaulay by Theorem 1.8. It follows that $H_{1}\left(\overline{I_{1} R}\right)$ and $H_{1}\left(\overline{J_{1} R}\right)$ are also Cohen-Macaulay by Lemma 1.5. In particular, $I_{1}$ and $J_{1}$ are syzygetic. Now the standard sequence (1.6) shows that $\operatorname{depth} \bar{R} /{\overline{I_{1} R}}^{2} \geq \operatorname{dim} R / I_{1} R-1$ and $\operatorname{depth} \bar{R} /{\overline{J_{1} R}}^{2} \geq \operatorname{dim} R / J_{1} R-1$. As $\overline{\mathcal{L}}^{2}={\overline{I_{1} R}}^{2} \cap{\overline{J_{1} R}}^{2}$, there is an exact sequence

$$
0 \rightarrow \bar{R} / \overline{\mathcal{L}}^{2} \rightarrow \bar{R} /{\overline{I_{1} R}}^{2} \oplus \bar{R} /{\overline{J_{1} R}}^{2} \rightarrow \bar{R} /{\overline{I_{1} R}}^{2}+{\overline{J_{1} R}}^{2} \rightarrow 0 .
$$

Notice that there are isomorphisms

$$
\begin{aligned}
\bar{R} /{\overline{I_{1} R}}^{2}+{\overline{J_{1} R}}^{2} & \cong R /\left(\underline{\alpha}, I_{1}^{2}\right) R+\left(\underline{\beta}, J_{1}^{2}\right) \\
& \cong k[[X]] /\left(\underline{\alpha}, I_{1}^{2}\right) \widehat{\otimes}_{k} k[[Y]] /\left(\beta, J_{1}^{2}\right),
\end{aligned}
$$

and thus by the previous estimates this module has depth $\geq \operatorname{dim} k[[X]] / I_{1}-1+$ $\operatorname{dim} k[[Y]] / J_{1}-1=\operatorname{dim} R / \mathcal{L}-2$. Thus by the previous sequence, one has that $\operatorname{depth} \bar{R} / \overline{\mathcal{L}}^{2} \geq \operatorname{dim} R / \mathcal{L}-1$. Finally, we are done using the sequence

$$
0 \rightarrow \oplus R / \mathcal{L} \rightarrow \mathcal{L} / \mathcal{L}^{2} \rightarrow \overline{\mathcal{L}} / \overline{\mathcal{L}}^{2} \rightarrow 0,
$$

which is exact as $\mathcal{L}$ is generated generically by the regular sequence $\underline{\alpha}, \underline{\beta}$ (as the link $\mathcal{L} \sim I R+J R$ is geometric).

To prove (b), it is enough to show by Lemma 1.5 that $\overline{\mathcal{L}}$ is not syzygetic, as $\mathcal{L}$ is generically a complete intersection. But $\overline{\mathcal{L}}=\overline{I_{1} R} \cdot \overline{J_{1} R}$, and as neither $I$ nor $J$ is Gorenstein, by Proposition $1.2 \mathrm{c}$, neither $\overline{I_{1} R}$ nor $\overline{J_{1} R}$ is principal. In this case, $\overline{\mathcal{L}}$, being a product, admits obvious quadratic relations on its generators, and hence is not syzygetic.

It remains to show (c). It is enough to show that $\overline{\mathcal{L}}$ is strongly Cohen-Macaulay. By Proposition $1.2 \mathrm{c}$ we have that $\overline{I_{1} R}=(a)$ is principal, and that $\overline{J_{1} R}$ satisfies the condition $G_{\infty}$. Thus $\overline{\mathcal{L}}=a \cdot \overline{J_{1} R}$ also satisfies $G_{\infty}$. Now $\overline{J_{1} R}$ is strongly Cohen-Macaulay, and thus from the approximation complexes ([7, proof of 4.6])

$$
\operatorname{depth} \bar{R} /{\overline{J_{1} R}}^{j} \geq \operatorname{dim} R / J_{1} R-j+1,
$$

for $j \geq 1$. On the other hand, $\overline{I_{1} R}$ is an almost complete intersection which is generically a complete intersection, and in particular is strongly Cohen-Macaulay and satisfies $G_{\infty}$. Thus by [14, 2.7], $\operatorname{depth} \bar{R} / \bar{I}_{1} R^{j} \geq \operatorname{dim} R / I_{1} R-1$, for $j \geq 1$. But now the analogue of the sequence of (a)

$$
0 \rightarrow \bar{R} / \overline{\mathcal{L}}^{j} \rightarrow \bar{R} /{\overline{I_{1} R}}^{j} \oplus \bar{R} /{\overline{J_{1} R}}^{j} \rightarrow \bar{R} /{\overline{I_{1} R}}^{j}+{\overline{J_{1} R}}^{j} \rightarrow 0
$$


shows that depth $\bar{R} / \overline{\mathcal{L}}^{i} \geq \operatorname{dim} R / \mathcal{L}-j+1$ for every $j$. The result now follows from $[27,2.13]$.

Lemma 2.5. Let $k$ be an algebraically closed field, let $A$ and $B$ be local $k$-algebras and let $B^{\prime}$ be a local $k$-algebra which is essentially a deformation of $B$. Assume that $A, B$ and $B^{\prime}$ are essentially of finite type over $k$. Then $\left(A \otimes_{k} B^{\prime}\right)_{M^{\prime}}$ is essentially a deformation of $\left(A \otimes_{k} B\right)_{M}$, where $M$ (respectively, $\left.M^{\prime}\right)$ is the maximal ideal $M=m_{A} \otimes B+A \otimes m_{B}$ (respectively, $\left.M^{\prime}=m_{A} \otimes B^{\prime}+A \otimes m_{B}\right)$.

Proof. If $B^{\prime}$ is a deformation of $B$, or if $B^{\prime}(X) \cong B(Y)$, then $\left(A \otimes_{k} B^{\prime}\right)_{M^{\prime}}$ and $\left(A \otimes_{k} B\right)_{M}$ have the same property. Suppose that $B^{\prime}=B_{p}$ is a localization of $B$ at a prime ideal $p$. Then as $k$ is algebraically closed, $m_{A} \otimes B+A \otimes p$ is a prime ideal in $A \otimes_{k} B$ and hence $\left(A \otimes B^{\prime}\right)_{M^{\prime}}$ is a localization of $(A \otimes B)_{M}$.

If $I \subset k[[X]]$ and $J \subset k[[Y]]$ are licci ideals, they are strongly nonobstructed and hence so is $I R+J R$ (by the Künneth formula) and its link $\mathcal{L}$. On the other hand, by Lemma 2.4b, if $I$ and $J$ are generically a complete intersection and not Gorenstein, then $\mathcal{L}$ is not strongly Cohen-Macaulay. The converse is given in the following theorem, which is one of our main results.

Theorem 2.6. Let $R$ be a regular local ring containing a field, and let $I$ and $J$ be two transversal licci $R$-ideals.

(a) If $I+J$ is generically a complete intersection, then the entire linkage class of $I+J$ is strongly Cohen-Macaulay if and only if $I$ or $J$ is Gorenstein.

(b) $I+J$ is licci only if $I$ or $J$ is a complete intersection.

Proof. We first reduce to the case when $R$ is complete. Clearly $I \widehat{R}$ and $J \widehat{R}$ are licci $\widehat{R}$-ideals, which are transversal by flatness, and their sum $I \widehat{R}+J \widehat{R}$ is still generically a complete intersection. Also, $I$ or $J$ is a complete intersection or is Gorenstein if and only if $I \widehat{R}$ or $J \widehat{R}$ has this property. Finally, an ideal is strongly Cohen-Macaulay if and only if it has this property after extension to $\widehat{R}$, and as this property is preserved under even linkage, it follows that the entire linkage class of $I+J$ is strongly Cohen-Macaulay if and only if $I \widehat{R}+J \widehat{R}$ has this property as well. Hence we may assume that $R$ is complete, i.e., $R=k\left[\left[x_{1}, \ldots, x_{n}\right]\right]$.

Now as $I$ and $J$ are transversal, $(R, I+J)$ has a deformation $(S, \widetilde{I}+\widetilde{J})$, where $S=$ $R\left[\left[y_{1}, \ldots, y_{n}\right]\right]$, and $\widetilde{I}$ and $\widetilde{J}$ are extended from $k\left[\left[x_{1}, \ldots, x_{n}\right]\right]$ and $k\left[\left[y_{1}, \ldots, y_{n}\right]\right]$, respectively. Indeed, one may take $\widetilde{I}=I S$ and $\widetilde{J}=\varphi(J)$, where $\varphi: k\left[\left[x_{1}, \ldots, x_{n}\right]\right] \rightarrow$ $k\left[\left[y_{1}, \ldots, y_{n}\right]\right]$ is the $k$-algebra homomorphism $\varphi\left(x_{i}\right)=y_{i}(1 \leq i \leq n)$. Now $\widetilde{I}$ and $\widetilde{J}$ are licci by Lemma $1.12, \widetilde{I}+\widetilde{J}$ is generically a complete intersection and similarly licci whenever $I+J$ is, and $\widetilde{I}$ and $\widetilde{J}$ is a complete intersection or Gorenstein if and only if $I$ or $J$ has this property. Let $I+J \sim L$ be any geometric link in $R$. Then by Lemma 1.12 there exists a $\operatorname{link} \widetilde{I}+\widetilde{J} \sim \widetilde{L}$ in $S$ such that $(S, \widetilde{L})$ is a deformation of $(R, L)$. As $L$ is generically a complete intersection, by Lemma $1.11 L$ is strongly Cohen-Macaulay if and only if $\widetilde{L}$ is. It follows that the entire linkage class of $I+J$ is strongly Cohen-Macaulay if and only if $\widetilde{I}+\widetilde{J}$ has the same property. We have thus reduced to the case where $R=k\left[\left[x_{1}, \ldots, x_{n}, y_{1}, \ldots, y_{n}\right]\right]$, and $I=\widetilde{I} R$ and $J=\widetilde{J} R$ are extended from $k\left[\left[x_{1}, \ldots, x_{n}\right]\right]$ and $k\left[\left[y_{1}, \ldots, y_{n}\right]\right]$, respectively.

Now suppose that $(S, \tilde{\tilde{I}})$ is a deformation of $\left(k\left[\left[x_{1}, \ldots, x_{n}\right]\right], \widetilde{I}\right)$, where $S$ is a complete local $k$-algebra. Then $\left(S\left[\left[y_{1}, \ldots, y_{n}\right]\right],(\tilde{\tilde{I}}, \widetilde{J})\right)$ is a deformation of $(R, I+J)$ (in other words, tensor products deform). As we have seen, all of our assumptions 
and conclusions are preserved as we pass from $\widetilde{I}$ and $\widetilde{J}$ to $\tilde{\tilde{I}}$ and $\widetilde{J}$. As $\widetilde{J}$ is licci it is strongly nonobstructed, and by a result of Herzog $([6,2.3])$ we may assume that $k\left[\left[y_{1}, \ldots, y_{m}\right]\right] / \widetilde{J}$ is a rigid $k$-algebra. In particular, we may assume that $\widetilde{I}$ and $\widetilde{J}$ are generically a complete intersection by $[13,6.1]$.

Now we prove (a). If the entire linkage class of $I+J$ is strongly Cohen-Macaulay, then the transversal link $\mathcal{L}(\widetilde{I}, \widetilde{J}) \sim I+J$ is strongly Cohen-Macaulay. By Lemma $2.4 \mathrm{~b}$, this implies that either $I$ or $J$ is Gorenstein. For the converse, we may assume that $I$ is Gorenstein. As $I+J$ is strongly Cohen-Macaulay by [9, 1.9], it is enough to show that $\mathcal{L}$ is strongly Cohen-Macaulay. Now as $J$ is licci, its entire linkage class is strongly Cohen-Macaulay. Thus by Lemma $2.4 \mathrm{c}$, it is enough to show that there exists a geometric link $J_{1}$ of $J$ such that $J_{1}+J / J$ satisfies $G_{\infty}$. It will even be sufficient to show this in some power series extension $R[[Z]]$ of $R$. The generic link $L_{1}(J) \subset R[Z]$ of $J$ is geometric, and by a result of Kustin and Miller [18, 4.2], the algebra $R[[Z]] / L_{1}(J) R[[Z]]$ is still rigid over $k$. In particular, $L_{1}(J) R[[Z]]$ satisfies $G_{\infty}([13,6.1])$ and thus $L_{1}(J) R[[Z]]+J R[[Z]] / J R[[Z]]$ satisfies $G_{\infty}$ by $[13,3.3]$. This proves that the entire linkage class of $I+J$ is strongly Cohen-Macaulay.

It remains to show (b). Let $\bar{k}$ denote the algebraic closure of $k$. Then the property of an $R$-ideal being licci ascends to $R \widehat{\otimes}_{k} \bar{k}$, while that of being a complete intersection will descend. Thus we may assume that $k$ is algebraically closed, and in particular is infinite.

Now $\widetilde{I} \subset k\left[\left[x_{1}, \ldots, x_{n}\right]\right]$ is licci, so there exists in particular a sequence of links in $k[[X]]$,

$$
I_{0}=\left(x_{1}, \ldots, x_{g}\right) \sim I_{1} \sim \cdots \sim I_{n}=\widetilde{I}
$$

(as all complete intersection of the same grade lie in the same linkage class). Consider a sequence of generic links $L_{i}\left(x_{1}, \ldots, x_{g}\right) \subset S_{i}, 1 \leq i \leq n$, where $S_{i}$ are polynomial extensions of $S_{0}=k[[X]]$, and put $S=S_{n}$. By Theorem 1.14, there exists a prime $p \in \operatorname{Spec} S$ such that $\left(S_{p}, L_{n}(\underline{x})_{p}\right)$ is a deformation of $(k[[X]], \widetilde{I})$. Similarly, there is a polynomial extension $T$ of $k[[Y]]$, containing a generic link $L_{m}\left(y_{1}, \ldots, y_{n}\right)$, and a prime $q \in \operatorname{Spec} T$ such that $\left(T_{q}, L_{m}(\underline{y})_{q}\right)$ is a deformation of $(k[[Y]], \widetilde{J})$.

Now the generic link $L_{n}\left(x_{1}, \ldots, x_{g}\right) \subset S$ (respectively, $L_{m}\left(y_{1}, \ldots, y_{h}\right) \subset T$ ) may be viewed as an extended ideal from the polynomial subring $k[X, Z] \subset S$ (respectively, $k[Y, U] \subset T$ ). Set $p^{\prime}=p \cap k[X, Z]$ and $q^{\prime}=q \cap k[Y, U]$. The faithfully flat morphisms

$$
\begin{aligned}
\left(k[X, Z] / L_{n}(\underline{x})\right)_{p^{\prime}} & \rightarrow\left(S / L_{n}(\underline{x})\right)_{p}, \\
\left(k[Y, U] / L_{m}(\underline{y})\right)_{q^{\prime}} & \rightarrow\left(T / L_{m}(\underline{y})\right)_{q}
\end{aligned}
$$

give a faithfully flat morphism of tensor products

$$
\begin{aligned}
& \left(\left(k[X, Z] / L_{n}(\underline{x})\right)_{p^{\prime}} \otimes_{k}\left(k[Y, U] / L_{m}(\underline{y})\right)_{q^{\prime}}\right)_{\left(p^{\prime}, q^{\prime}\right)}
\end{aligned}
$$

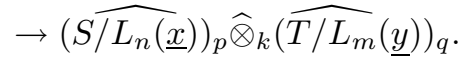

Moreover, the latter algebra is a deformation of $k[[X]] / \widetilde{I} \widehat{\otimes}_{k} k[[Y]] / \widetilde{J} \cong R / I+J$. Now as $k$ is an infinite field, the property that a Noetherian local $k$-algebra is a quotient of a regular local $k$-algebra by a licci ideal is independent of any presentation (as can be seen using [12, 2.12], and [20, 2.11]). We have thus reduced to the following situation: $\widetilde{R}$ and $\widetilde{S}$ are regular local rings essentially of finite type over $k$, and $\widetilde{I} \subset \widetilde{R}$, 
$\widetilde{J} \subset \widetilde{S}$ are licci ideals which are not complete intersections. We must show that $\left(\widetilde{R} / \widetilde{I} \otimes_{k} \widetilde{S} / \widetilde{J}\right)_{M}$ is not licci, where $M$ is the maximal ideal $m_{\widetilde{R} / \widetilde{I}} \otimes \widetilde{S} / \widetilde{J}+\widetilde{R} / \widetilde{I} \otimes m_{\widetilde{S} / \widetilde{J}}$.

By part (a), we may assume that $\widetilde{I}$ is Gorenstein. Now Theorem 1.15 implies that $\left(P[W] / P f_{4}(W)\right)_{\left(m_{P}, W\right)}$, where $P$ is a regular local ring and $W$ is a generic alternating $5 \times 5$ matrix, is essentially a deformation of $\widetilde{R} / \widetilde{I}$, while $\widetilde{S} / \widetilde{J}$ has essentially a deformation either $\left(P[W] / P f_{4}(W)\right)_{\left(m_{P}, W\right)}$ or $\left(P[V] / I_{2}(V)\right)_{\left(m_{P}, V\right)}$, with $V$ a generic $2 \times 3$ matrix. Moreover, by the proof of $[25,2.6], P$ may be taken to be essentially of finite type over $k$ as well. As the property of being licci is preserved under essentially a deformation (Lemma 1.12 and $[20,2.12]$ ), by Lemma 2.5 we may assume that $\widetilde{R} / \widetilde{I}=\left(P[W] / P f_{4}(W)\right)_{\left(m_{P}, W\right)}=\widetilde{A}$ and $\widetilde{S} / \widetilde{J}=\widetilde{R} / \widetilde{I}$, or $\left(P[V] / I_{2}(V)\right)_{\left(m_{P}, V\right)}=\widetilde{B}$. On the other hand, as the ideals $P f_{4}(W)$ and $I_{2}(V)$ are extended from polynomial $k$-subalgebras, there are faithfully flat morphisms

$$
\left(k[W] / P f_{4}(W) \otimes_{k} k[W] / P f_{4}(W)\right)_{M^{\prime}} \rightarrow\left(\widetilde{A} \otimes_{k} \widetilde{A}\right)_{M}
$$

and

$$
\left(k[W] / P f_{4}(W) \otimes_{k} k[V] / I_{2}(V)\right)_{M^{\prime}} \rightarrow\left(\widetilde{A} \otimes_{k} \widetilde{B}\right)_{M} .
$$

Hence by faithfully flat descent, we are reduced to showing that $A \otimes_{k} A$ and $A \otimes_{k} B$ are not licci (after localization at the maximal ideal generated by the variables) where $A=k[W] / P f_{4}(W)$ and $B=k[V] / I_{2}(V)$. We will show this by computing their homogeneous resolutions.

Let $\widetilde{R}=k[W]$ and $\widetilde{S}=k[V]$. Then $A$ has a homogeneous resolution over $\widetilde{R}$

$$
0 \rightarrow \widetilde{R}(-5) \rightarrow \widetilde{R}^{5}(-3) \rightarrow \widetilde{R}^{5}(-2) \rightarrow \widetilde{R} \rightarrow A \rightarrow 0
$$

while $B$ has a homogeneous resolution over $\widetilde{S}$

$$
0 \rightarrow \widetilde{S}^{2}(-3) \rightarrow \widetilde{S}^{3}(-2) \rightarrow \widetilde{S} \rightarrow B \rightarrow 0
$$

Hence if $R=\widetilde{R} \otimes_{k} \widetilde{R}$ and $S=\widetilde{R} \otimes_{k} \widetilde{S}$, then $A \otimes_{k} A$ has a homogeneous resolution

$$
0 \rightarrow R(-10) \rightarrow \cdots \rightarrow R^{10}(-2) \rightarrow R \rightarrow A \otimes_{k} A \rightarrow 0
$$

and $A \otimes_{k} B$ has a homogeneous resolution

$$
0 \rightarrow S^{2}(-8) \rightarrow \cdots \rightarrow S^{8}(-2) \rightarrow S \rightarrow A \otimes_{k} B \rightarrow 0 .
$$

In the latter case, note that $8=(2+3-1) \cdot 2$, and in the former case, $10=(3+3-1) \cdot 2$. Thus by using the criterion $[11,5.8]$, it follows that $\left(A \otimes_{k} A\right)_{M}$ and $\left(A \otimes_{k} B\right)_{M}$ are not licci. This completes the proof of the theorem.

Corollary 2.7. Let $R$ be a regular local ring containing a field, let $I$ and $J$ be two transversal licci $R$-ideals which are not complete intersections, and let $K=I+J$. Then $K$ is strongly Cohen-Macaulay, strongly nonobstructed, $\operatorname{Tor}_{i}^{R}\left(R / K, \omega_{R / K}\right)$ is Cohen-Macaulay for every $i$, and $K$ is not licci.

Proof. Theorem 2.6 implies that $K$ is not licci. As $I$ and $J$ are licci, they are strongly Cohen-Macaulay, and by [4], $\operatorname{Tor}_{i}^{R}\left(R / I, \omega_{R / I}\right)$ and $\operatorname{Tor}_{i}^{R}\left(R / J, \omega_{R / J}\right)$ are Cohen-Macaulay for every $i$. As $I / I^{2} \otimes_{R / I} \omega_{R / I} \cong \operatorname{Tor}_{i}^{R}\left(R / I, \omega_{R / I}\right)$, the rest follows from the Künneth formula (cf., e.g., [9, pp. 746-748]).

We conclude this section with the simplest generic examples. 
Example 2.8. Let $k$ be a field, let $X$ and $Y$ be generic $2 \times 3$ matrices, let $U$ and $V$ be generic alternating $5 \times 5$ matrices, and let $R=k[[X, Y, U, V]]$. The following ideals are not licci:

(a) $I_{2}(X)+I_{2}(Y)$ is a grade 4 , deviation 2, type 4 strongly Cohen-Macaulay and strongly nonobstructed ideal.

(b) $I_{2}(X)+P f_{4}(U)$ is a grade 5 , deviation 3 , type 2 strongly nonobstructed ideal whose entire linkage class is strongly Cohen-Macaulay.

(c) $P f_{4}(U)+P f_{4}(V)=K$ is a grade 6 , deviation 4, Gorenstein strongly CohenMacaulay ideal with $\operatorname{Tor}_{\bullet}^{R}(R / K, R / K)$ Cohen-Macaulay.

\section{SumS OF LINKED IDEALS}

In this section we study the sum $K=I+J$ of two geometrically linked CohenMacaulay ideals $I$ and $J$ in a local Gorenstein ring. The link $I \sim J$ being geometric, one knows that $K$ is a Gorenstein ideal with grade $K=$ grade $I+1([21,1.4])$. A result of Ulrich [26, 2.1] guarantees that $K$ is licci whenever $I$ is. But following his lead in [26], one may still investigate how certain properties, such as being strongly nonobstructed or strongly Cohen-Macaulay, pass from $I$ and $J$ to $I+J$.

We will need some auxiliary results involving the depth of certain relevant modules.

Lemma 3.1. Let $R$ be a local Gorenstein ring, and let $I$ and $J$ be geometrically linked Cohen-Macaulay $R$-ideals, which are not both grade one principal ideals.

$$
\operatorname{depth} I J=\operatorname{depth}\left(I \otimes \omega_{R / I}\right) / \tau \text {. }
$$

Proof. We may assume that $g=$ grade $I>0$, else both modules in question vanish. Let $\underline{\alpha}$ be the regular sequence defining the link $I \sim J$. Tensoring the exact sequence

$$
0 \rightarrow \omega_{R / I} \rightarrow R /(\underline{\alpha}) \rightarrow R / J \rightarrow 0
$$

with $R / I$ gives an exact sequence

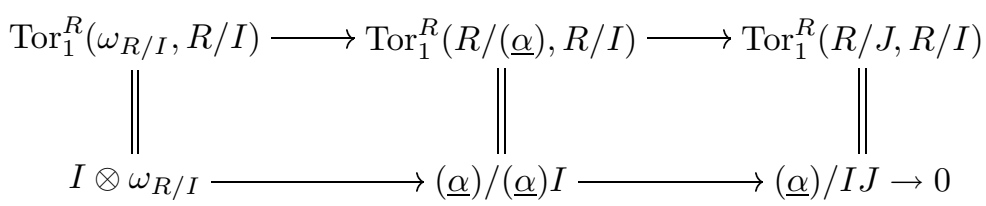

$\operatorname{Now}(\underline{\alpha}) /(\underline{\alpha}) I \cong(\underline{\alpha}) \otimes_{R} R / I \cong(\underline{\alpha}) /(\underline{\alpha})^{2} \otimes_{R /(\underline{\alpha})} R / I \cong(R /(\underline{\alpha}))^{g} \otimes_{R /(\underline{\alpha})} R / I \cong$ $(R / I)^{g}$, and $\underline{\alpha}$ generates $I$ generically as the link $I \sim J$ is geometric. Thus one obtains an exact sequence

$$
0 \rightarrow\left(I \otimes \omega_{R / I}\right) / \tau \rightarrow(R / I)^{g} \rightarrow(\underline{\alpha}) / I J \rightarrow 0 .
$$

The result follows once we show that $R / I J$ is not Cohen-Macaulay: for then $\operatorname{depth}\left(I \otimes \omega_{R / I}\right) / \tau=\operatorname{depth}(\underline{\alpha}) / I J+1=\operatorname{depth} R / I J+1=\operatorname{depth} I J$.

Suppose $R / I J$ is Cohen-Macaulay. Then $I J$ is unmixed, and it follows that $I J=I \cap J=(\underline{\alpha})$. If " - " denotes reduction modulo $\left(\alpha_{1}, \ldots, \alpha_{g-1}\right)$, then one has that the product $I J=\left(\bar{\alpha}_{g}\right)$ is principal. It follows that $I$ and $J$ are invertible, and hence principal as $R$ is local. Thus $I=\left(\alpha_{1}, \ldots, \alpha_{g-1}, a\right)$ and $J=\left(\alpha_{1}, \ldots, \alpha_{g-1}, b\right)$ for some $a \in I$ and $b \in J$. But then $I=\left(\alpha_{1}, \ldots, \alpha_{g-1}, a\right) \subset(I J, a)$ and $I=(a)$ by Nakayama's Lemma. Similarly, $J=(b)$. As $I$ and $J$ are assumed not both principal, we conclude that $R / I J$ is not Cohen-Macaulay. 
The next result is a variant of a formula of [26] (which follows immediately from its proof) equating the depth of $H_{1}(I)$ with that of the second symmetric power of the canonical module of a link of $I$.

Lemma 3.2 ([26, 3.1]). Let $R$ be a local Gorenstein ring and let $I$ be a CohenMacaulay $R$-ideal. Then one of the following conditions holds:

(a) depth $H_{1}(I)=\operatorname{depth} S_{2}(I)$

(b) $H_{1}(I)$ is Cohen-Macaulay and $\operatorname{depth} S_{2}(I) \geq \operatorname{dim} R / I+1$.

We are now prepared to state one of our main results. It significantly generalizes $[26,3.11]$, which gives conditions for a sum of geometrically linked ideals to be syzygetic and strongly nonobstructed.

Theorem 3.3. Let $R$ be a local Gorenstein ring, let $I$ and $J$ be two geometrically linked Cohen-Macaulay ideals, and let $K=I+J$.

(a) depth $K / K^{2}=\min \left\{\operatorname{depth} I / I^{2}, \operatorname{depth} J / J^{2}, \operatorname{depth} I \otimes \omega_{R / I} / \tau-1, \operatorname{dim} R / K\right\}$

(b) depth $H_{1}(K)=\min \left\{\operatorname{depth} H_{1}(I), \operatorname{depth} H_{1}(J), \operatorname{depth} I \otimes \omega_{R / I}, \operatorname{dim} R / K\right\}$.

Proof. We first prove (a). If $I$ is a complete intersection, then so is $K$ by Proposition $1.2 \mathrm{c}$, and the result is clear. Thus we may assume $I$ is not a complete intersection. Let $\underline{\alpha}$ be the regular sequence defining the link $I \sim J$. As $(\underline{\alpha}) /(\underline{\alpha}) I \cong(R / I)^{g}$, $\operatorname{depth} R /(\underline{\alpha}) I=\operatorname{dim} R / I$ and similarly $\operatorname{depth} R /(\underline{\alpha}) J=\operatorname{dim} R / J$. But as $\underline{\alpha}$ generates $I$ and $J$ generically, $(\underline{\alpha}) I \subset I^{2} \cap I J \subset I^{2} \cap(\underline{\alpha})=\underline{\alpha} I$ and $\underline{\alpha} J \subset J^{2} \cap I K \subset$ $J^{2} \cap\left(I^{2}, \underline{\alpha}\right) \subset J^{2} \cap\left(I^{2} \cap J, \underline{\alpha}\right)=J^{2} \cap(\underline{\alpha})=\underline{\alpha} J$, hence all containments are equalities. It follows that there are exact sequences

$$
\begin{aligned}
& 0 \rightarrow(\underline{\alpha}) I \rightarrow I^{2} \oplus I J \rightarrow I K \rightarrow 0, \\
& 0 \rightarrow(\underline{\alpha}) J \rightarrow I K \oplus J^{2} \rightarrow K^{2} \rightarrow 0 .
\end{aligned}
$$

Using Lemma 3.1, (a) now follows by a depth chase.

To prove (b), we reduce to the grade 0 case. Let "- " denote reduction modulo the linking sequence $\underline{\alpha}$ Then the depth of $H_{1}$ of any of $I, J$ or $K$ is unchanged passing to $I, J$ or $K$ by Lemma 1.5, while there is an exact sequence

$$
0 \rightarrow \omega_{R / I}^{g} \rightarrow I \otimes \omega_{R / I} \rightarrow I \otimes \omega_{\bar{R} / \bar{I}} \rightarrow 0 .
$$

Thus the formula of (b) is unchanged by factoring out $(\underline{\alpha})$, and we may thus assume that $g=0$.

Now $K=I \oplus J \cong I \oplus \omega_{R / I}$, so

$$
S_{2}(K) \cong S_{2}(I) \oplus S_{2}(J) \oplus\left(I \otimes \omega_{R / I}\right) .
$$

As $g=0$, Lemma 3.2 implies that depth $H_{1}(I)=\operatorname{depth} S_{2}(I)$ and depth $H_{1}(I)=$ depth $S_{2}(J)$. Thus (b) will follow from (3.4) once we show that depth $H_{1}(K)=$ depth $S_{2}(K)$. If this is not the case, by Lemma 3.2 we would have that depth $S_{2}(K)$ $=\operatorname{dim} R$, and in particular $S_{2}(K)$ would be torsion free. But then (3.4) would imply that $I \otimes \omega_{R / I} \cong I \otimes J=0$, and thus that $I=0$ or $J=0$. This contradiction completes the proof.

Corollary 3.5 (cf. $[26,3.11]$ ). Let $R$ be a local Gorenstein ring and let $K=I+J$ be a sum of two geometrically linked Cohen-Macaulay R-ideals.

(a) $K$ is strongly nonobstructed if and only if

$$
\operatorname{depth} R / I^{2} \geq \operatorname{dim} R / I-1, \quad \operatorname{depth} R / J^{2} \geq \operatorname{dim} R / J-1,
$$

and $\left(I \otimes \omega_{R / I}\right) / \tau$ is Cohen-Macaulay. 
(b) Suppose that I satisfies $\left(C I_{1}\right)$. Then $K$ is syzygetic if and only if $I$ and $J$ are syzygetic, and $I \otimes \omega_{R / I}$ is torsion free.

Proof. As $K$ is Gorenstein, $K \otimes \omega_{R / K} \cong K / K^{2}$, so $K$ is strongly nonobstructed if and only if the conormal module $K / K^{2}$ is Cohen-Macaulay. Thus (a) follows immediately from Theorem 3.3(a).

For (b), suppose first that $I$ and $J$ are syzygetic and $I \otimes_{R} \omega_{R / I}$ is torsion free. Then $H_{1}(I)$ and $H_{1}(J)$ are torsion free, and then so is $H_{1}(K)$ by Theorem 3.3(b). As $K$ is generically a complete intersection, since $I$ satisfies $\left(C I_{1}\right)$, it follows that $K$ is syzygetic. (This would follow from $[26,3.10]$ even if $I$ does not satisfy $\left(C I_{1}\right)$.) Conversely, suppose that $K$ is syzygetic. Then $H_{1}(K)$ is torsion free and by Theorem 3.3(b), $H_{1}(I)$ and $I \otimes \omega_{R / I}$ are torsion free as $I$ satisfies $\left(C I_{1}\right)$. As $I$ is generically a complete intersection, in particular $I$ is syzygetic. As $J$ is also generically a complete intersection, it is enough to show that $H_{1}(J)$ is also torsion free, which by Theorem 3.3(b) is enough to check locally in codimension one. But if $p \in V(J)$ and $\operatorname{dim}(R / J)_{p}=1$, then $I_{p} \sim J_{p}$, so $J_{p}$ is an almost complete intersection, which is well-known to be syzygetic. It follows that $J$ is syzygetic.

Remark 3.6. One may use Theorem 3.3 to contrast the properties that $H_{1}$ is CohenMacaulay with that of being strongly nonobstructed. For perfect Gorenstein ideals, the difference first appears in grade 5 . Indeed, a perfect Gorenstein ideal of grade $\leq 2$ is a complete intersection, of grade 3 is licci ([33]), and of grade 4 is strongly nonobstructed if and only if $H_{1}$ is Cohen-Macaulay ([31]). An example where the properties do not coincide is given in $[26,5.3]$ (or see the next result). That these two properties coincide for perfect Gorenstein ideals if grade 4 implies by Theorem 3.3 some relations on the depths of the modules $H_{1}(I), H_{1}(J)$, and $I \otimes_{R} \omega_{R / I}$ for perfect ideals $I$ and $J$ of grade 3 , which are (geometrically) linked. A more complete picture can be seen by the work of Vasconcelos [29]; we mention only one result: If $I$ and $J$ are two linked perfect ideals of grade 3 , then $H_{1}(I)$ is Cohen-Macaulay if and only if $H_{1}(J)$ is Cohen-Macaulay.

We now combine the results of this and the previous section to construct some examples. They are similar in nature to the examples of [26], whose properties were verified with computer assistance. These examples inspired much of the work of this paper.

Proposition-Example 3.7. let $I \subset k\left[\left[x_{1}, \ldots, x_{n}\right]\right]$ and $J \subset k\left[\left[y_{1}, \ldots, y_{m}\right]\right]$ be two non-Gorenstein licci ideals that satisfy $\left(C I_{1}\right)$, and let $\mathcal{L}$ be a geometric transversal link of $I$ and $J$ in $R=k\left[\left[x_{1}, \ldots, x_{n}, y_{1}, \ldots, y_{m}\right]\right]$. Then $K=I R+J R+\mathcal{L}$ is a strongly nonobstructed Gorenstein R-ideal which is not syzygetic.

Proof. Let $A=I R+J R$, so that $K$ is the sum of the geometrically linked ideals $A \sim \mathcal{L}$. By Lemma $2.4 \mathrm{~b}, \mathcal{L}$ is not syzygetic. As $A$ satisfies $\left(C I_{1}\right)$, Corollary $3.5 \mathrm{~b}$ implies that $K$ is not syzygetic. On the other hand, $A$ is strongly nonobstructed and strongly Cohen-Macaulay $([9,1.9])$ and thus the exact sequence (1.6) implies that $\operatorname{depth} R / A^{2} \geq \operatorname{dim} R / A-1$. Finally, by Lemma $2.4 \mathrm{a}$, $\operatorname{depth} R / \mathcal{L}^{2} \geq \operatorname{dim} R / \mathcal{L}-1$. Thus $K$ is strongly nonobstructed by Corollary 3.5a.

Example 3.8. Let $I=\left(x_{1} x_{2}, x_{2} x_{3}, x_{3} x_{4}\right)$ and $J=\left(y_{1} y_{2}, y_{2} y_{3}, y_{3} y_{4}\right)$ in $R=$ $k\left[\left[x_{1}, \ldots, x_{4}, y_{1}, \ldots, y_{4}\right]\right]$. Then $I$ and $J$ are grade 2 perfect $R$-ideals, and hence are licci. One may view $I$ and $J$ as edge ideals of the graph [32]: 
1926

MARK R. JOHNSON

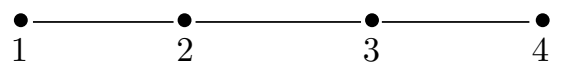

FigURE 1

Linking via the outer edges produces a transversal link $\mathcal{L}=\left(x_{1} x_{2}, x_{3} x_{4}, y_{1} y_{2}, y_{3} y_{4}\right.$, $\left.x_{1} y_{1}, x_{1} y_{4}, x_{4} y_{1}, x_{4} y_{4}\right)$. By Lemma $2.4, \mathcal{L}$ is a perfect $R$-ideal of grade 4 , deviation 4 and type 2 , which is strongly nonobstructed, satisfies $\operatorname{depth} R / \mathcal{L}^{2} \geq \operatorname{dim} R / \mathcal{L}-1$, but is not syzygetic. Moreover, $\mathcal{L}$ may be viewed as the ideal of the following graph:

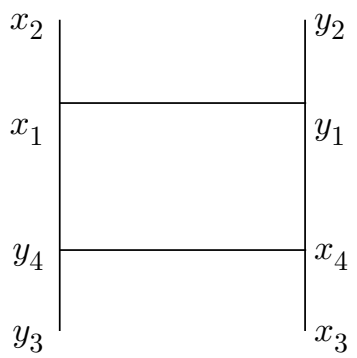

FiguRE 2

Now Proposition 3.7 implies that $K=I+J+\mathcal{L}=\left(x_{1} x_{2}, x_{2} x_{3}, x_{3} x_{4}, y_{1} y_{2}, y_{2} y_{3}, y_{3} y_{4}\right.$, $\left.x_{1} y_{1}, x_{1} y_{4}, x_{4} y_{1}, x_{4} y_{4}\right)$ is a Gorenstein ideal of grade 5 and deviation 5 which is strongly nonobstructed and not syzygetic. The ideal $K$ is the ideal of the graph

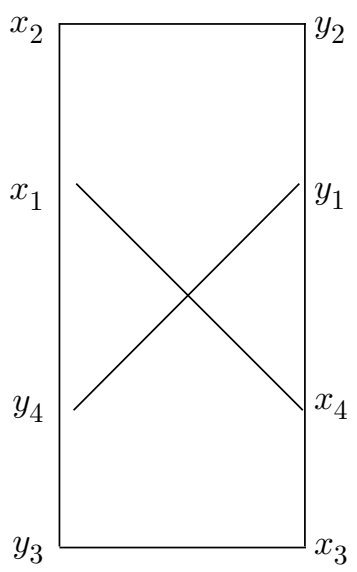

Figure 3

We give another way to obtain the previous example $K$ as a sum of linked ideals. It will give a reduced perfect ideal of grade 4 whose entire linkage class is strongly Cohen-Macaulay but whose twisted conormal module has torsion. (A similar example $[26,5.3 \mathrm{a}]$ has grade 5 .)

Example 3.9. Consider the edge ideal $I$ of the tree 


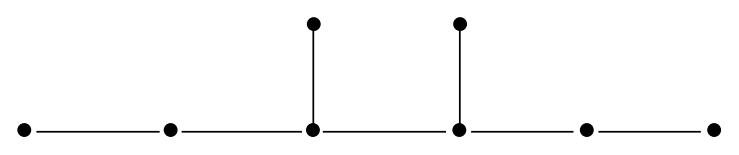

FIGURE 4

It is easily seen that $I$ is a perfect ideal of grade 4 (e.g., by [32]), deviation 3 and type 3 . Moreover by [23, 3.11], $I$ is strongly Cohen-Macaulay and linking via the four outer edges gives an ideal $J$ with the same graph, and thus is also strongly CohenMacaulay. It follows that the entire linkage class of $I$ is strongly Cohen-Macaulay. However, $K=I+J$ is a Gorenstein ideal of grade 5 generated by ten quadrics. It is easy to see that it is the ideal of Example 3.8. Thus $K$ is not syzygetic and hence $I \otimes \omega_{R / I}$ has torsion by Corollary 3.5b. On the other hand, $K$ is strongly nonobstructed, by Corollary 3.5a it follows that $\left(I \otimes \omega_{R / I}\right) / \tau$ is Cohen-Macaulay.

We conclude with an application which proves part of a claim made in $[27,2.11]$ and $[16,3.6]$, which was first verified by computer.

Example 3.10. Let $k$ be a field, $X$ be a generic alternating $5 \times 5$ matrix, and $Y$ a generic $5 \times 1$ matrix, let $R=k[X, Y]$ (possibly localized at the irrelevant maximal ideal), and let $I=P f_{4}(X)+I_{1}(X Y)$ be the ideal generated by the $4 \times 4$ Pfaffians of $X$ and the entries of the product matrix $X Y$.

This example has been recently considered in the context of algebra structures on the minimal resolution ([24]). It is well-known that $R / I$ is the associated graded algebra of the ideal $P f_{4}(X)$, and that it is Gorenstein $([8,2.2])$. We will show that $I / I^{2}$ is Cohen-Macaulay, and that $H_{1}(I)$ is not Cohen-Macaulay. In fact, we will show that $I$ is doubly linked to a deformation of the ideal $K$ of Example 3.8. The claim then follows from Theorems 1.8 and 1.9, Lemma 1.11, and [14, 2.2].

We have $I=\left(f_{1}, \ldots, f_{5}, l_{1}, \ldots, l_{5}\right)$, where $f_{i}$ denotes the Pfaffian obtained by deleting the $i$-th row and column of $X$, and $l_{i}$ is the product of the $i$-th row of $X$ with $Y$. Write $X=\left(x_{i j}\right)_{1 \leq i, j \leq 5}, Y=\left(Y_{i}\right)_{1 \leq i \leq 5}$.

As $X$ is alternating, $Y^{t}(X Y)=0$, so there is a relation

$$
y_{1} l_{1}+\cdots+y_{5} l_{5}=0
$$

on $l_{1}, \ldots, l_{5}$. In particular, $y_{5} \in\left(l_{1}, \ldots, l_{4}\right): l_{5}$. On the other hand, it is well known that $R / I_{1}(X Y)$ is the Rees algebra of $P f_{4}(X)$, and thus is a Cohen-Macaulay domain $([8,2.2])$. It then follows (and in any event is easy to see directly) that $l_{1}, \ldots, l_{4}$ is a regular sequence. Now as $\left(l_{1}, \ldots, l_{5}\right)$ defines the Rees algebra of $\left(f_{1}, \ldots, f_{5}\right)$, in particular $X \cdot(\underline{f})^{t}=0$, and hence $Y^{t} X(\underline{f})^{t}=0$. Thus we obtain another relation

$$
f_{1} l_{1}+\cdots+f_{5} l_{5}=0
$$

on $l_{1}, \ldots, l_{5}$. It follows that $I$ is not syzygetic. In particular, this relation gives $f_{5} \in\left(l_{1}, \ldots, l_{4}\right): l_{5}$. Thus we have that the link $\left(l_{1}, \ldots, l_{4}\right): l_{5}$ contains the ideal $\left(l_{1}, \ldots, l_{4}, y_{5}, f_{5}\right)$. However the latter ideal is easily seen to be a hyperplane section of the generic grade 3 Gorenstein ideal of $4 \times 4$ Pfaffians, and in particular is prime. The previous remarks then imply that $l_{1}, \ldots, l_{4}, f_{1}$ is a regular sequence. Thus we may consider the link $\left(l_{1}, \ldots, l_{4}, f_{1}\right): I$. To compute this ideal we need a few more 
obvious relations:

$$
\begin{aligned}
& y_{1} f_{5}-y_{5} f_{1}=x_{34} l_{2}-x_{24} l_{3}+x_{23} l_{4}, \\
& y_{1} f_{4}-y_{4} f_{1}=x_{35} l_{2}-x_{25} l_{3}+x_{23} l_{5}, \\
& y_{1} f_{3}-y_{3} f_{1}=x_{45} l_{2}-x_{25} l_{4}+x_{24} l_{5}, \\
& y_{1} f_{2}-y_{2} f_{1}=x_{45} l_{3}-x_{35} l_{4}+x_{34} l_{5} .
\end{aligned}
$$

After multiplying these equations by $y_{5}$ and using (3.11), we find that $y_{1} y_{5} \in J=$ $\left(l_{1}, \ldots, l_{4}, f_{1}\right)$. In fact, $J=\left(l_{1}, \ldots, l_{4}, f_{1}, y_{1} y_{5}\right)$. Indeed, as $J /\left(l_{1}, \ldots, l_{4}, f_{1}\right)$ is cyclic (Proposition 1.2c), it would be enough by degree reasons to show that $J$ contains no linear form. This may be readily checked by specializing sufficiently many of the variables. (One could also see this from the homogeneous resolution of $R / I$, which has the form

$$
0 \rightarrow R(-8) \rightarrow \cdots \rightarrow R^{10}(-2) \rightarrow R \rightarrow R / I \rightarrow 0 .
$$

By mapping cone construction [21], $J$ must have a resolution

$$
0 \rightarrow R^{5}(-8) \rightarrow \cdots \rightarrow R^{6}(-2) \rightarrow R \rightarrow R / J \rightarrow 0
$$

and thus contains no linear form.)

Now it is easy to see that $l_{1}, l_{3}, l_{4}, f_{1}, y_{1} y_{5}$ is a regular sequence contained in $J$, so we may form the link

$$
\begin{aligned}
K= & \left(l_{1}, l_{3}, l_{4}, f_{1}, y_{1} y_{5}\right): J, \\
& \left(l_{1}, l_{3}, l_{4}, f_{1}, y_{1} y_{5}\right): l_{2} .
\end{aligned}
$$

We may similarly as above compute the generators of $K$. Clearly $y_{1} y_{2} \in K$ by equation (3.11), while $x_{34} y_{5}, x_{34} y_{2} \in K$ by using (3.12) and (3.15), respectively. On the other hand, by (3.13) and (3.14) $x_{23} y_{2}-x_{35} y_{5}$ and $x_{24} y_{2}-x_{45} y_{5}$ belong to $K$. Thus $K$ also contains $l_{3}+\left(x_{23} y_{2}-x_{35} y_{5}\right)=-x_{13} y_{1}+x_{34} y_{4}$, and $l_{4}+\left(x_{24} y_{2}-x_{45} y_{5}\right)=-x_{14} y_{1}-x_{34} y_{3}$. Hence $K$ contains ten quadrics $l_{1}, f_{1}, y_{1} y_{5}, y_{1} y_{2}, x_{34} y_{5}, x_{34} y_{2}, x_{23} y_{2}-x_{35} y_{5},-x_{13} y_{1}+x_{34} y_{4}, x_{24} y_{2}-x_{45} y_{5}, x_{14} y_{1}+$ $x_{34} y_{3}$. But it is now obvious that the ideal generated by these quadrics specializes (e.g., via $x_{12}=x_{13}=x_{15}=x_{24}=x_{25}=x_{35}=y_{3}=0$ ) to the ideal (also called $K$ ) of Example 3.8. In particular, the former ideal is Cohen-Macaulay, and it then follows that $K$ is precisely the ideal generated by these quadrics. Thus we have shown that $I$ is doubly linked to a deformation of the ideal of Example 3.8, which proves the claim.

We make one final remark about the last example. In [27] and [16] the ideal $I$ is claimed to have the additional surprising property that $R / I^{3}$ is Cohen-Macaulay (this again was only verified by computer). More surprisingly perhaps is, this is false if $\operatorname{char} k=2$. Presumably this is related to the fact that the homogeneous resolution of $R / I$ differs when $k$ has characteristic 2. Indeed, by [24] if char $k \neq 2$, the minimal resolution has the form

$$
0 \rightarrow R(-8) \rightarrow R^{10}(-6) \rightarrow R^{16}(-5) \rightarrow R^{16}(-3) \rightarrow R^{10}(-2) \rightarrow R \rightarrow R / I \rightarrow 0,
$$

while in char 2 it is

$$
\begin{aligned}
0 \rightarrow & R(-8) \rightarrow R^{10}(-6) \rightarrow \bigoplus_{R^{16}(-5)}^{R(-4)} \rightarrow \bigoplus_{R(-4)}^{R^{16}(-3)} \\
& \rightarrow R^{10}(-2) \rightarrow R \rightarrow R / I \rightarrow 0 .
\end{aligned}
$$




\section{REFERENCES}

1. R. Apéry, Sur les courbes de première espèce de l'espace de trois dimensions, C. R. Acad. Sci. Paris 220 (1945), 271-272. MR 7:170c

2. M. Auslander, Modules over unramified regular local rings, Illinois J. Math. 5 (1961), 632-647. MR 86i: 16032

3. R.-O. Buchweitz, Contributions à la thèorie des singularités, Thesis, l'Université de Paris, 1981.

4. R.-O. Buchweitz and B. Ulrich, Homological properties which are invariant under linkage, preprint.

5. F. Gaeta, Détermination de la chaine syzygétique des idéaux matriciels parfaits et son application à la postulation de leurs variétés algébriques associées, C. R. Acad. Sci. Paris 234 (1954), 1833-1835. MR 13:978a

6. J. Herzog, Deformationen von Cohen-Macaulay Algebren, J. Reine Angew. Math. 318 (1980), 83-105. MR 81m:13012

7. J. Herzog, A. Simis, and W. V. Vasconcelos, Koszul homology and blowing-up rings, Commutative Algebra, Proceedings, Trento, 1981, Lecture Notes in Pure and Appl. Math. 84, Marcel Dekker, 1983, pp. 79-169. MR 84a:13002

8. C. Huneke, Linkage and Koszul homology of ideals, Amer. J. Math. 104 (1982), 1043-1062. MR 84f: 13019

9. C. Huneke, Strongly Cohen-Macaulay schemes and residual intersections, Trans. Amer. Math. Soc. 277 (1983), 739-763. MR 84m:13023

10. C. Huneke and B. Ulrich, Divisor class groups and deformations, Amer. J. Math. 107 (1985), 1265-1303. MR 87f: 13010

11. C. Huneke and B. Ulrich, The structure of linkage, Ann. of Math. 126 (1987), 277-334. MR 88k:13020

12. C. Huneke and B. Ulrich, Algebraic linkage, Duke Math. J. 56 (1988), 415-429. MR 89e:13023

13. C. Huneke and B. Ulrich, Residual intersections, J. Reine Angew. Math. 390 (1988), 1-20. MR 89j: 13024

14. C. Huneke and B. Ulrich, Powers of licci ideals, in Commutative Algebra (Berkeley), Math. Sci. Res. Int. Publ. 15, Springer, 1989, pp. 339-346. MR 90i:13008

15. C. Huneke and B. Ulrich, Local properties of licci ideals, Math. Z. 211 (1992), 129-154. MR 93j: 13018

16. M. Johnson and B. Ulrich, Artin-Nagata properties and Cohen-Macaulay associated graded rings, Compositio Math. 103 (1996), 7-29. MR 97f:13006

17. A. Kustin and M. Miller, A general resolution for grade four Gorenstein ideals, Manuscripta Math. 35 (1981), 221-269. MR 83g:14026

18. A. Kustin and M. Miller, Deformation and linkage of Gorenstein algebras, Trans. Amer. Math. Soc. 284 (1984), 501-533. MR 85k:13015

19. S. Lichtenbaum, On the vanishing of Tor in regular local rings, Illinois J. Math. 10 (1966), 220-226. MR 32:5688

20. E. Lopez, Licci Gorenstein ideals of deviation two, Ph.D. thesis, Michigan State University (1988).

21. C. Peskine and L. Szpiro, Liaison des variétés algébriques, Invent. Math. 26 (1974), 271-302. MR 51:526

22. A. Simis and W. V. Vasconcelos, The syzygies of the conormal module, Amer. J. Math. 103 (1981), 203-224. MR 82i:13016

23. A. Simis, W. V. Vasconcelos, and R. Villarreal, On the ideal theory of graphs, J. Algebra 167 (1994), 389-416. MR 95e:13002

24. H. Srinivasan, A grade five Gorenstein algebra with no minimal algebra resolutions, J. Algebra 179 (1996), 362-379. MR 96j:13012

25. B. Ulrich, Theory and applications of universal linkage, in Commutative Algebra and Combinatorics, M. Nagata and H. Matsumura (eds.), Adv. Studies in Pure Math. 11, North-Holland, Amsterdam, 1987, pp. 285-301. MR 89b:13001

26. B. Ulrich, Sums of linked ideals, Trans. Amer. Math. Soc. 318 (1990), 1-42. MR 90f:13012

27. B. Ulrich, Artin-Nagata properties and reductions of ideals, Contemp. Math. 159 (1994), 373-400. MR 95a:13017

28. B. Ulrich, Parafactoriality and small divisor class groups, in preparation. 
29. W. V. Vasconcelos, Koszul homology and the structure of low codimensional Cohen-Macaulay ideals, Trans. Amer. Math. Soc. 301 (1987), 591-613. MR 88i:13031

30. W. V. Vasconcelos, Arithmetic of Blowup Algebras, London Math. Soc. Lecture Note Series, 1993. MR 95g:13005

31. W. V. Vasconcelos and R. Villarreal, On Gorenstein ideals of codimension four, Proc. Amer. Math. Soc. 98 (1986), 205-210. MR 87k:13041

32. R. Villarreal, Cohen-Macaulay graphs, Manuscripta Math. 66 (1990), 277-293. MR 91b:13031

33. J. Watanabe, A note on Gorenstein rings of embedding codimension three, Nagoya Math. J. 50 (1973), 227-232. MR 47:8526

Department of Mathematical Sciences, University of Arkansas, Fayetteville, ARKansas 72701

E-mail address: mark@math.uark.edu 